\title{
Cinémas
}

Revue d'études cinématographiques

Journal of Film Studies

\section{Le jazz à la télévision française dans les années 1950 : du didactisme des premières représentations aux expérimentations de Jean-Christophe Averty} Jazz on French Television in the 1950s: From the Didacticism of
the Early Years to the Experimentation of Jean-Christophe
Averty

\section{Gilles Mouëllic}

Volume 26, numéro 2-3, printemps 2016

La télévision... selon Jean-Christophe Averty

URI : https://id.erudit.org/iderudit/1039366ar

DOI : https://doi.org/10.7202/1039366ar

Aller au sommaire du numéro

Éditeur(s)

Cinémas

ISSN

1181-6945 (imprimé)

1705-6500 (numérique)

Découvrir la revue

Citer cet article

Mouëllic, G. (2016). Le jazz à la télévision française dans les années 1950 : du didactisme des premières représentations aux expérimentations de Jean-Christophe Averty. Cinémas, 26(2-3), 51-71.

https://doi.org/10.7202/1039366ar
Résumé de l'article

Très présent à la radio et dans la presse françaises après la Seconde Guerre mondiale, le jazz va conquérir peu à peu la télévision à partir de 1955. À la recherche du jazz, première série régulière sur le sujet, créée par Maurice Blettery et réalisée, pour l'écrasante majorité des émissions, par Jean-Christophe Averty, révèle une tension entre deux ambitions didactiques : l'une qui fait du jazz un prétexte pour aborder des « sujets de société », l'autre qui marque une volonté d'étudier la musique pour elle-même et d'affirmer le jazz comme témoignage d'un nouvel équilibre entre le " savant » et le " populaire ". Alors que les premières émissions sont construites autour d'une succession souvent maladroite d'extraits de longs métrages et de discours d'experts reconnus, Averty s'émancipe progressivement des contraintes éditoriales de la série pour mettre en images la musique et explorer les moyens propres à la télévision. À partir de la fin de la décennie, il prend en charge de nouvelles émissions, dont Modern Jazz at Studio 4, dans lesquelles il privilégie la performance des musiciens et fait du jazz un terrain d'expérimentation idéal pour inventer un univers plastique que l'on peut qualifier, bien avant les oeuvres de Nam June Paik, d’art vidéo. 


\title{
Le jazz à la télévision française dans les années 1950 : du didactisme des premières représentations aux expérimentations de Jean-Christophe Averty
}

\section{Gilles Mouëllic}

\begin{abstract}
RÉSUMÉ
Très présent à la radio et dans la presse françaises après la Seconde Guerre mondiale, le jazz va conquérir peu à peu la télévision à partir de 1955 . À la recherche du jazz, première série régulière sur le sujet, créée par Maurice Blettery et réalisée, pour l'écrasante majorité des émissions, par Jean-Christophe Averty, révèle une tension entre deux ambitions didactiques: l'une qui fait du jazz un prétexte pour aborder des "sujets de société", l'autre qui marque une volonté d'étudier la musique pour ellemême et d'affirmer le jazz comme témoignage d'un nouvel équilibre entre le «savant» et le "populaire». Alors que les premières émissions sont construites autour d'une succession souvent maladroite d'extraits de longs métrages et de discours d'experts reconnus, Averty s'émancipe progressivement des contraintes éditoriales de la série pour mettre en images la musique et explorer les moyens propres à la télévision. À partir de la fin de la décennie, il prend en charge de nouvelles émissions, dont Modern Jazz at Studio 4, dans lesquelles il privilégie la performance des musiciens et fait du jazz un terrain d'expérimentation idéal pour inventer un univers plastique que l'on peut qualifier, bien avant les œuvres de Nam June Paik, d'art vidéo.
\end{abstract}

L'objet de cette étude est de comprendre la nature et les enjeux de la présence du jazz dans les années d'expérimentation de la télévision en France, avec pour hypothèse que les caractéristiques propres au jazz vont faire de cette musique un lieu 
d'exploration du devenir possible du nouveau médium, tout autant qu'un lieu d'affirmation de l'univers créatif de JeanChristophe Averty. Avant 1955, ainsi que le rapporte Philippe Lavat (2011) dans un article sur l'apparition du jazz à la télévision, les programmes publiés ne font guère état du jazz, si ce n'est une Jam Session ${ }^{1}$ au mois de décembre 1950, une émission de la série Plaques tournantes intitulée "Paris, capitale du jazz ${ }^{2}$ " diffusée en novembre 1952, plus quelques moments dans deux autres séries appelées Musique pour vous et Musique télévisée. Peu de choses en réalité avant le début de l'année 1955, quand commence une série d'une vingtaine d'émissions (de durées assez variables mais proches en moyenne de quarante minutes) intitulée $\grave{A}$ la recherche $d u$ jazz, programmée sans véritable régularité jusqu'en 1959, année où elle est supplantée, semble-t-il, par Jazz Memories de Jean-Christophe Averty, personnage incontournable de toute réflexion sur les relations entre la télévision et le jazz. L'absence de choix éditorial affirmé de la série $A$ la recherche $d u$ jazz, si elle témoigne des tâtonnements de ces premières années, autorise une remarquable diversité d'approches dont le seul point commun est le jazz, décliné à l'envi par Maurice Blettery ${ }^{3}$, auteur et voix de la série avec la complicité d'Averty, présent sur la quasi-totalité des émissions.

Cette première série d'émissions régulières permet d'analyser comment la télévision va à la fois revendiquer une ambition didactique et concevoir ses propres codes de représentation. Le jazz, musique née d'une histoire noire de l'Amérique, inventée dans quelques villes du sud des États-Unis au tournant du $\mathrm{XIX}^{\mathrm{e}}$ siècle avant de s'imposer au monde occidental comme un modèle d'émancipation politique et artistique, est susceptible de toucher un public populaire tout en servant de matière à un discours savant. Les nombreux extraits de films puisés dans l'histoire du cinéma américain privilégient un jazz spectacle en mêlant sans hiérarchie numéros de comédies musicales, images des big bands de Duke Ellington et apparitions burlesques de Fats Waller. Mais ces extraits cohabitent très vite avec des performances filmées par la télévision, accompagnées de commentaires d'experts destinés à faire comprendre les véritables spécificités de la musique de jazz, légitimée par de supposées flatteuses 


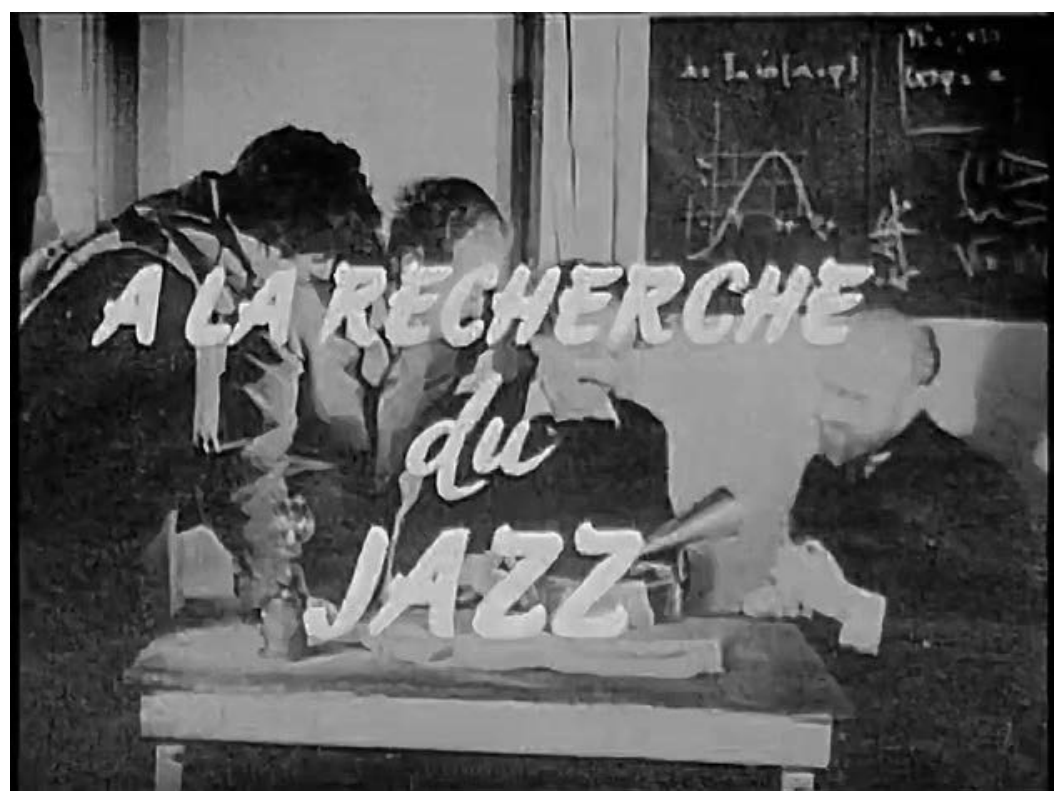

Fig. 1. Un générique de la série $\grave{A}$ la recherche du jazz.

comparaisons avec la musique dite "classique». En réalisant la plupart des émissions de cette série, Jean-Christophe Averty expérimente à partir du jazz et de l'enregistrement en direct les techniques de la télévision pour inventer peu à peu un langage spécifique, précurseur de ce qui deviendra l'art vidéo.

\section{Une histoire noire du jazz}

Les deux premières émissions de la série, diffusées les 13 janvier et 22 février 1955, témoignent d'une certaine prudence devant une musique peu connue, associée dans l'imaginaire du grand public à la Libération et aux caves de Saint-Germain-desPrés, voire aux comédies musicales américaines ${ }^{4}$. Maurice Blettery remonte le Mississippi, depuis la Louisiane jusqu'au Minnesota, avec des arrêts à Memphis et à Chicago, pour donner au spectateur quelques repères sur les origines à la fois musicales et géographiques du jazz. Il raconte sur fond d'images documentaires et de moments musicaux préexistants comment New York fut à son tour conquise par les orchestres, ce voyage étant accompagné d'une présentation des courants qui ont "fait» le jazz, à savoir le blues, le ragtime, le New Orleans, le swing ou le be-bop.

Le jazz à la télévision française dans les années 1950 : du didactisme des premières représentations aux expérimentations de Jean-Christophe Averty 
Cette entrée en matière répond à l'ambition didactique de la télévision naissante, et le jazz se révèle un sésame fort utile pour traiter une multitude de sujets qui seront déclinés sans souci d'une véritable structure, contrairement à ce que laisse penser cet intérêt premier pour la chronologie et la terminologie. Si d'autres émissions feront allusion à cet ancrage américain, les arguments seront très souvent associés à des problèmes de société analysés à partir du jazz, de son histoire et de ses diverses représentations. L'émission À la recherche du jazz du 30 décembre 1956, intitulée "Plainte noire", exceptionnellement réalisée par Igor Barrère et présentée par l'acteur de théâtre Jean Bolo, est tout à fait symptomatique de cette tendance. Elle commence par des images de rock censées évoquer la révolte de la jeunesse contre la bourgeoisie, suivies d'une évocation de James Dean, mort quelques mois plus tôt (en septembre 1955), présenté comme musicien de jazz amateur jouant de la clarinette et de la batterie. Entre le rock, le rhythm and blues et le jazz, entre la révolte "musicale» des jeunes blancs et les fondements de la musique noire dans les souffrances de l'esclavage puis de la ségrégation, les enchainements sont aussi inattendus qu'improbables. Cette volonté peu précautionneuse d'associer Blancs et Noirs dans de semblables tragédies culmine avec le montage de la voix de Billie Holiday interprétant le douloureux Strange Fruit sur des images évoquant bien plus les camps de concentration nazis que les plantations du sud des États-Unis. Cette surprenante association constitue un exemple parmi d'autres de l'absence de rigueur d'auteurs que le manque de moyens oblige à puiser sans grand scrupule dans des banques d'images et de sons. On perçoit aussi dans ces approximations la volonté de la télévision de mêler plusieurs niveaux de lecture et de compréhension, en prenant appui sur un passé reconstruit par une voix over au ton très docte et par un montage d'images préexistantes puisées dans diverses sources pas toujours identifiables, parmi lesquelles d'innombrables extraits de films.

\section{Les ressources du cinéma}

La télévision est, on le sait, grande consommatrice de cinéma dès ses premières années d'existence, et les films sont aussi des ressources essentielles pour des séries comme $\grave{A}$ la recherche $d u$ 
jazz, dont certaines émissions sont composées d'une suite parfois ininterrompue de séquences de longs métrages, comme cette célébration de l'Amérique du spectacle intitulée «Music Party à New York" (29 décembre 1957) où se succèdent des extraits de comédies musicales avec Fred Astaire notamment, des fêtes somptueuses dans des décors à la Cedric Gibbons, des performances de Fats Waller, de Louis Armstrong ou de Gene Krupa issues de films jamais référencés, le tout dans un joyeux désordre ponctué de feux d'artifice célébrant l'arrivée de la nouvelle année. L'émission "Chicago Université d'Al Capone (22 septembre 1957), titre éloquent s'il en est, est construite sur un modèle voisin avec une évocation des années de la prohibition, prétexte une fois encore à une mise bout à bout de passages de nombreux films dont les liens avec le jazz se limitent à la présence récurrente d'un petit orchestre de New Orleans faisant danser les quelques clients d'un honky-tonk enfumé, ou à celle, plus réjouissante, d'un pianiste de boogie-woogie continuant imperturbablement à marteler son clavier au milieu de rafales de mitraillettes. Mais la musique s'impose peu à peu comme le sujet de cette émission, Jean-Christophe Averty ne ratant pas l'occasion de sélectionner de très belles images de sa période de prédilection de l'histoire du jazz, celle des années 1920 et de l'avènement du New Orleans: on découvre Louis Armstrong, Bix Beiderbecke, Fats Waller, Earl Hines ou Bessie Smith — dans un extrait de St. Louis Blues (Dudley Murphy, 1929) — ou encore quelques plans de Charlot boxeur (The Champion, 1915) destinés à rappeler les rencontres mythiques entre le cinéma muet et les jazzmen dans les salles des quartiers noirs. Moins soucieuse de la qualité musicale mais tout aussi cinématographique, l'émission intitulée "Le couple» (28 juillet 1957) est elle aussi constituée d'un montage d'extraits dont le but est de représenter une diversité de situations mettant en présence un homme et une femme, séquences dont la bande originale a un rapport parfois lointain avec le jazz: on reconnaît La blonde et moi (The Girl Can't Help It, Frank Tashlin, 1956), Riz amer (Riso amaro, Giuseppe De Santis, 1949), La poupée de chair (Baby Doll, Elia Kazan, 1956), Antoine et Antoinette (Jacques Becker, 1947) ou Manèges (Yves Allégret, 1950), entre quelques cartoons.

Le jazz à la télévision française dans les années 1950 : du didactisme des premières représentations aux expérimentations de Jean-Christophe Averty 
Cette présence très importante du cinéma dans $A$ la recherche $d u$ jazz donne heureusement lieu à des documents bien plus précieux, comme cette émission dont l'invité unique est Michel Legrand (26 janvier 1958), bien avant sa collaboration avec Jacques Demy, ou cette autre intitulée «Venise» $\left(1957^{\circ}\right)$, organisée autour d'un long et passionnant échange avec Roger Vadim qui vient de réaliser dans la Sérénissime Sait-on jamais? (1957). Entre deux extraits du film, Vadim, face caméra, explique avec beaucoup de pédagogie le choix très novateur du Modern Jazz Quartet pour composer la musique, détaillant la méthode de travail avec le compositeur John Lewis, analysant les rapports entre musique et images à partir de quelques séquences. Il précise que certaines d'entre elles ont donné lieu à des improvisations du quartet directement sur les images projetées en studio, plusieurs mois, donc, avant l'enregistrement de la fameuse bande originale d'Ascenseur pour l'échafaud (Louis Malle, 1958). Cette façon de parler du cinéma à partir du jazz, avec une attention singulière au processus de création et à la parole du cinéaste évoquant son travail, annonce déjà ce que seront les meilleures émissions consacrées au cinéma, dont bien sûr la fameuse série Cinéastes de notre temps (qui débutera en 1964). Parmi les formidables entretiens d'A la recherche $d u$ $j a z z$, on retiendra encore une conversation de plus de dix-huit minutes avec Jean Cocteau évoquant ses surprenants choix musicaux pour Orphée (1950) et les raisons de son amour très précoce du jazz. Dans cette même émission du 24 avril 1956, intitulée "Pourquoi le jazz?» et portant sur les rapports entre le jazz et le cinéma, Jean Painlevé, au milieu d'étranges machines, raconte pendant près de dix minutes le scandale provoqué par les sonorités jungle de l'orchestre de Duke Ellington sur les images de son Vampire (1945). On retiendra enfin, dans l'émission intitulée "Le jazz et la jeunesse" (26 juillet 1956), un entretien avec Jacques Becker à propos de la jeunesse de SaintGermain-des-Prés, dont il est devenu un expert convoité après la réalisation de son Rendez-vous de juillet (1949). 


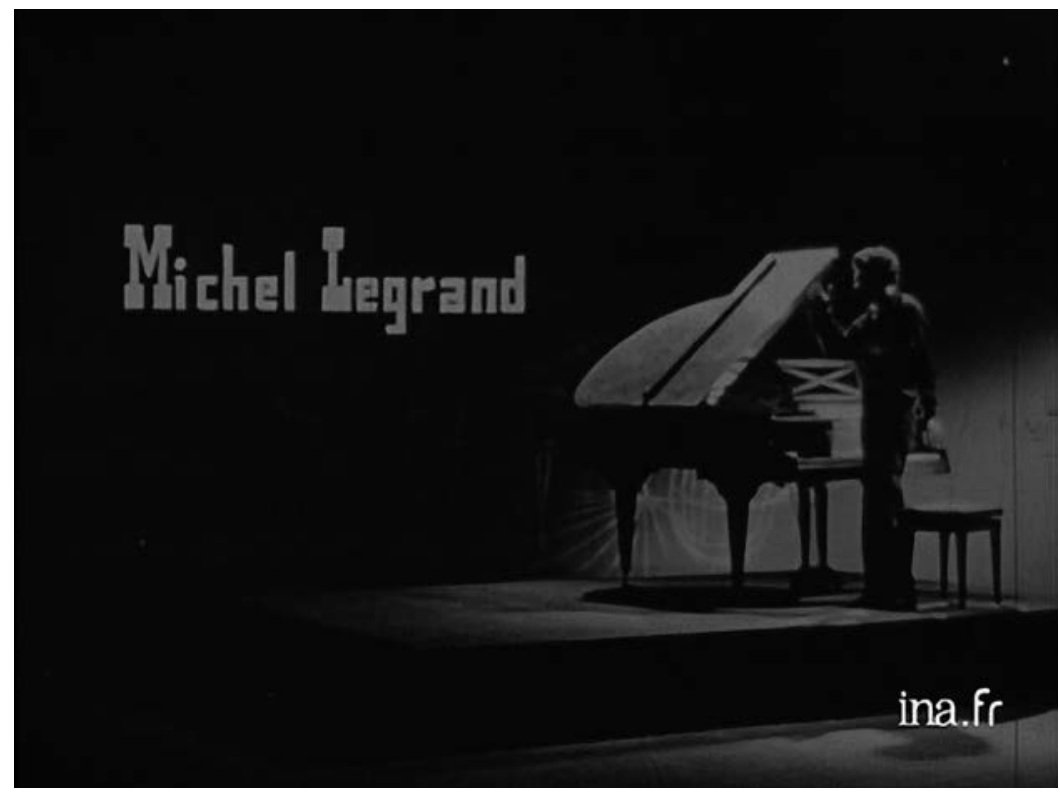

Fig. 2. Générique de l'émission $\grave{A}$ la recherche $d u$ jazz consacrée à Michel Legrand (Jean-Christophe Averty, 26 janvier 1958).

\section{Le jazz, musique savante}

Les émissions qui se libèrent des images préexistantes pour proposer des formes nouvelles et prendre en compte avec plus ou moins d'audace certaines spécificités du nouveau média sont parmi les plus passionnantes de la série. Le jazz devient alors le matériau idéal pour développer des propositions qui seront reprises plus tard dans d'autres programmes, notamment ceux de la télévision scolaire. Loin des facilités compilatoires évoquées plus haut, et dans la même veine que les entretiens avec Vadim, Cocteau ou Painlevé, plusieurs émissions revendiquent une haute ambition intellectuelle en prenant le parti de convoquer devant la caméra musiciens et musicologues. L'importance du jazz dans la vie intellectuelle en France n'est pas nouvelle, et se trouve renforcée par le goût de l'Amérique de l'après-guerre et soutenue par la présence de nombreux musiciens américains de premier plan. Certains s'installent dans l'Hexagone pour plusieurs années, tels le pianiste Bud Powell et le batteur Kenny Clarke, ce dernier invité remarqué d'À la recherche du jazz. Maurice Blettery puise dans le réservoir constitué par les revues

Le jazz à la télévision française dans les années 1950 : du didactisme des premières représentations aux expérimentations de Jean-Christophe Averty 
et les nombreuses émissions de radio un certain nombre d'experts parmi lesquels l'arrangeur et musicien Christian Chevallier, le compositeur et musicologue André Hodeir, le poète et écrivain Robert Goffin ou le critique et éditeur MichelClaude Jalard, tous très conscients de leur rôle de "passeurs". L'analyse aussi austère que remarquable proposée par Jalard du style du Modern Jazz Quartet, dont l'«originalité [...] ne réside pas dans la matière, mais dans une rigueur absolument inégalée de la mise en ouvre ${ }^{7}$ ", ou Cocteau affirmant que le jazz cool "correspond aux algèbres de Mozart, de Bach et de Schoenberg ${ }^{8}$ " ne sont que des exemples parmi bien d'autres de ces manifestations d'une volonté de prendre très (parfois trop) au sérieux une musique que les films américains ont emprisonnée dans l'image rassurante et réductrice de l'entertainment et des grands orchestres. En replaçant le jazz, même de façon maladroite on l'a vu, dans une histoire qui est aussi celle de la présence tragique des Noirs sur le territoire américain, et en utilisant les outils d'analyse propres à la musique savante pour tenter d'en dévoiler quelques clés de compréhension, les auteurs de ces émissions contribuent à donner au jazz d'inattendues lettres de noblesse. Mais ce désir d'en faire une musique convenable constitue aussi la limite de l'exercice, tant est contestable cette vision très occidentalo-centrée privilégiant la dimension compositionnelle, ce que l'on a appelé alors le "troisième courant", fondé sur des compromis possibles entre la musique "classique» et le jazz. L'émission du 23 juin 1957, intitulée "Quand le jazz s'inspire de la musique des XVII et XVIII ${ }^{e}$ siècles", marque une forme d'aboutissement de cette lecture très partisane où la soumission à la toute-puissance de l'écriture semble le seul chemin que puisse emprunter le jazz pour conquérir le monde de l'art. Cette tentative de légitimation du jazz par une mise en scène du discours intellectuel privilégiant la forme écrite répond à un projet plus vaste d'appropriation européenne d'une musique qui prétend désormais à l'universalité. Mais elle n'est pas sans rapport avec les besoins de légitimation de la télévision elle-même, dont un des credo semble être de réconcilier le populaire et le savant. Le jazz, dont la France a défendu très tôt l'originalité et la complexité contre une image exotique imposée par le cinéma 


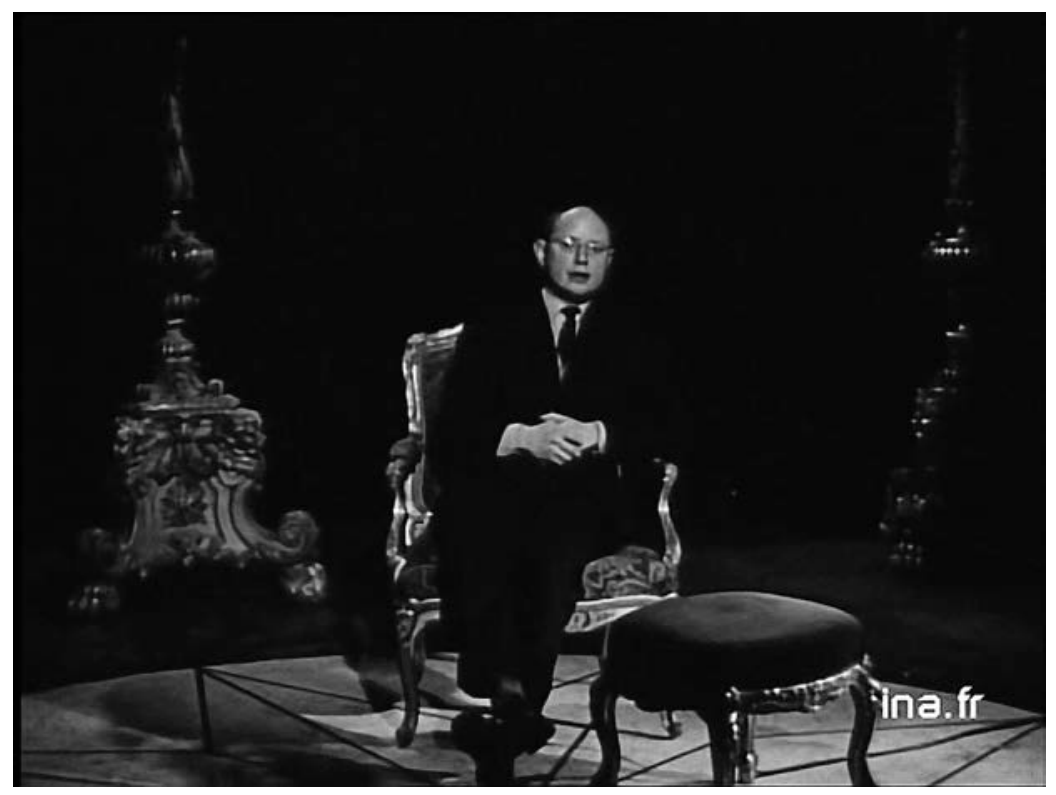

Fig. 3. Mise en scène de la parole savante: Michel-Claude Jalard parle du Modern Jazz Quartet dans l'émission À la recherche du jazz du 23 juillet 1957, réalisée par Jean-Christophe Averty.

et les revues de music-hall, est alors un modèle très fécond de ces possibles croisements.

\section{Le jazz parmi les arts}

Si imposer le jazz au sein de la première télévision nécessite un excès de sérieux qui ne lui sied guère, cela n'empêche pas certains des auteurs d'À la recherche $d u$ jazz d'exploiter sa part d'irrévérence et d'impertinence, sous l'évidente influence d'Averty, peu soupçonnable de complaisance à l'égard d'un anoblissement du jazz qui passerait par la musique dite "savante». L'émission intitulée "Spectacle insolite» (28 août 1956), consacrée notamment aux rapports entre le jazz et le surréalisme, explore avec humour et un brin d'insolence les relations entre la création artistique et les diverses drogues. Un psychiatre très sérieux, qui répond au nom de docteur Katz (!), rend compte de la diversité des effets des stupéfiants sur le comportement humain, avant de conclure non sans précautions que ces effets ne sont en rien bénéfiques pour les artistes. Mezz

Le jazz à la télévision française dans les années 1950 : du didactisme des premières représentations aux expérimentations de Jean-Christophe Averty 
Mezzrow, consommateur avéré de ces divers produits, jazzman et auteur avec Bernard Wolfe du célèbre essai biographique $\mathrm{La}$ rage de vivre (Really the Blues, 1946), témoigne lui aussi de cette influence peu concluante sur le jeu des musiciens, propos confirmés par un très long extrait de L'homme au bras d'or (The Man with the Golden Arm, 1955) d'Otto Preminger, où le batteur Frankie Machine (Frank Sinatra) échoue lamentablement à une audition après avoir repris ses mauvaises habitudes de consommateur d'héroïne. Mais cette surprenante émission est surtout l'occasion de mettre en scène les rencontres poétiques entre le jazz et les autres arts, rencontres dont la diversité constitue une des richesses de la série dans son ensemble. Tout en rendant un hommage appuyé aux dessins sur pellicule de Norman McLaren et d'un de ses émules français, Albert Pierru (redécouvert il y a peu ${ }^{9}$ ), la télévision met en scène avec ses propres moyens des correspondances visuelles à base de montages, de surimpressions ou d'effets de répétition: des danseurs improvisent sur un solo de saxophone, une chanteuse semble se promener dans les rues sombres de Paris dans une ambiance très "film noir" ("L'école française du jazz moderne", 10 février 1957), une improvisation virtuose de Charlie Parker est mise en images par une succession de tableaux et de dessins symbolistes, une danseuse improvise sur fond de reproductions de Salvador Dalí ("Spectacle insolite») ou de jardins à la française ("Music Party», $1958{ }^{10}$ ). Plusieurs créations bien plus abstraites sont inspirées par le jazz moderne d'André Hodeir ou du Modern Jazz Quartet à nouveau, avec des variations virtuoses sur les nuances de noir et de blanc, tels ces deux pieds, l'un noir l'autre blanc, se promenant dans la profondeur de champ, cet emboîtement de décors d'un petit théâtre investi par un danseur habillé en Pierrot ou ce quatuor de danseurs et de danseuses évoluant dans un décor très mobile qui semble lui aussi entrer en danse: il faudrait citer tous les numéros de ce très beau "Music Party» consacré à la danse.

On reconnaît bien vite certaines pratiques d'Averty qui, sans négliger la mise en scène des nombreux entretiens, toujours sobre et élégante, semble bien plus à son aise dans une exploration ludique des possibilités du médium. Si certains montages 
d'extraits de films signalent déjà un intérêt pour les collages, pour les rythmes plastiques déterminés par exemple par des enchaînements de gestes ou de lumière, les expérimentations sont bien plus novatrices dans ces moments autonomes où Averty met en images en toute liberté un morceau de jazz. À la recherche $d u$ jazz permet de comprendre la diversité des influences d'Averty puisées dans le travail sur le noir et blanc des grands chefs opérateurs du cinéma américain, dans les magnificences des comédies musicales chorégraphiées par Busby Burkeley, dans la fluidité des numéros dansés de Fred Astaire ou dans les nombreuses représentations de la musique noire célébrée par Dudley Murphy dans Black and Tan (1929) ou par Gjon Mili dans Jammin' the Blues (1944). Une chorégraphie avec deux mains en gros plans, l'une gantée de blanc l'autre de noir, à laquelle répond une danse des pieds sur fond noir; une mise en images du jazz moderne d'André Hodeir par des robots, suivie d'une danse de petites boules sur une composition de Christian Chevallier (deux moments de «L'école française du jazz moderne»); un mélange, dans "Music Party à New York », de néons clignotants, d'extraits de comédies musicales et de numéros survoltés de danseurs à claquettes noirs: Averty met à l'épreuve de la télévision son imagination débridée. Il compose de nouvelles formes en puisant dans une connaissance insatiable de l'histoire des arts et une dévorante curiosité pour les rencontres entre la culture noire et le cinéma à l'origine de cette photogénie du jazz qui sera au fondement de son langage d'artiste. Consciemment ou non, à partir des libres représentations d'À la recherche du jazz, il expérimente les outils qui vont lui permettre d'inventer un art nouveau, une tele music ${ }^{11}$, ainsi que le suggère le générique de Modern Jazz at Studio 4 du 6 avril 1961. Les dessins de ce générique rappellent ceux d'À la recherche $d u$ jazz, mais le travail graphique sera rapidement bien plus inventif, comme dans ce générique de Blue Note Parade (6 avril 1963) qui évoque les fameuses pochettes de disques de la firme Blue Note (figures 4 et 5), des pochettes devenues vivantes, les incrustations de visages étant ici en mouvement. 

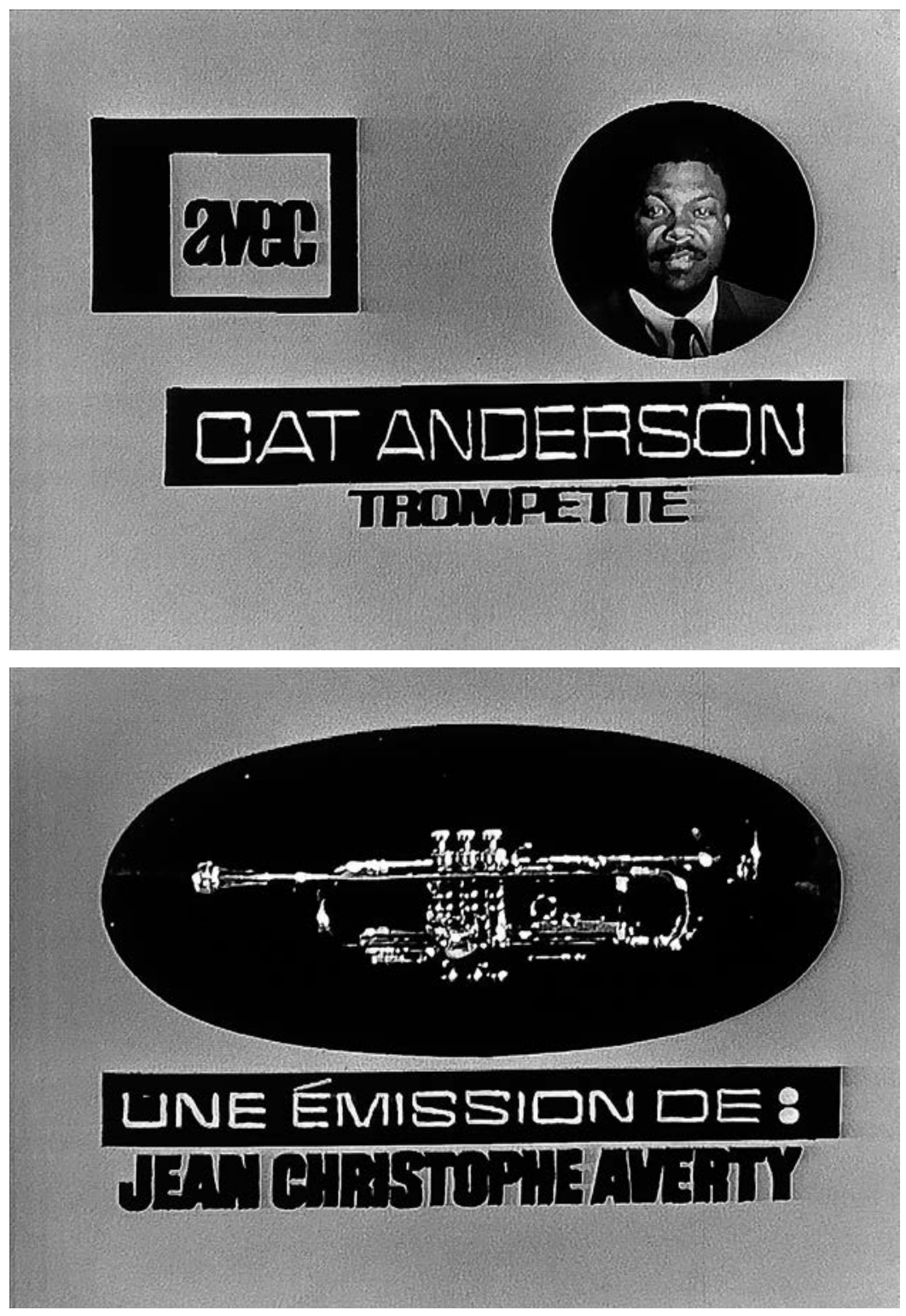

Fig. 4 et 5. Générique de Blue Note Parade (Jean-Christophe Averty, 6 avril 1963).

5. Mettre en scène l'enregistrement (la question du direct) Si les prémices de cette tele music sont repérables dans les émissions où Averty tente de trouver des équivalents ou des prolongements plastiques du jazz dans la danse, le cinéma 
d'animation ou les montages d'œuvres picturales, ce sont bien les moments où il travaille directement la matière musicale qui annoncent les œuvres à venir. Contrairement à ce que l'on pourrait penser, la série $\grave{A}$ la recherche $d u$ jazz n'est jamais diffusée en direct et, nous l'avons vu, son contenu ne répond guère à cet impératif de captation de performances associé aux premières années de la télévision. Cependant, l'enregistrement des musiciens au travail est bien un enjeu important, ainsi que le montre l'émission du 27 octobre 1957, intitulée "On enregistre». Pendant plus de quarante minutes, Blettery et Averty documentent les différentes étapes de l'enregistrement dans un studio parisien d'un disque du quartet du pianiste et compositeur Christian Chevallier, avec Kenny Clarke à la batterie, Pierre Michelot à la contrebasse et Roger Guérin à la trompette. Filmer le travail des musiciens, c'est aussi filmer le travail de la télévision, c'est filmer un dispositif de captation dans une mise en scène de cette "esthétique du direct" qu'André Bazin théorise au même moment dans plusieurs textes parfaitement analysés par Gilles Delavaud (2003 et 2007). Si Averty prend grand soin dans chacune des émissions qu'il réalise de découvrir de nouvelles manières de donner à voir la musique, c'est qu'il comprend que le jazz dans sa dimension improvisée est la musique idéale d'une télévision qui affirme peu à peu son identité dans sa capacité à capter des événements. Le jazz est un moment de création collective dont l'implication dans le présent de la performance suppose une virtuosité qui fascine Averty et que l'on retrouve symboliquement dans le direct télévisuel dont il deviendra très vite un maître. Le studio d'enregistrement filmé à plusieurs caméras est une manière de montrer à la fois la fabrication d'un disque et la fabrication des images, de célébrer cette modernité figurée par la modernité du dispositif télévisuel et par la modernité du jazz lui-même ${ }^{12}$.

Loin des tentatives de faire du jazz une musique écrite, très présentes on l'a vu dans $\grave{A}$ la recherche du jazz, le choix d'Averty dans les émissions dont il aura le contrôle dès 1958 sera de célébrer l'improvisation en filmant les concerts à plusieurs caméras dans les théâtres, puis dans les festivals qui se multiplient alors

Le jazz à la télévision française dans les années 1950 : du didactisme des premières représentations aux expérimentations de Jean-Christophe Averty 
dans toute la France. Mais quelle que soit la virtuosité de ces réalisations, c'est en studio qu'Averty conçoit des dispositifs qui lui permettent d'improviser à son tour à partir des images des musiciens, sans jamais remettre en cause l'autonomie de la musique, la continuité de la performance. Dans les formidables émissions qui se succèdent alors, parmi lesquelles Modern Jazz at Studio 4, Averty met au point en un temps record une synthèse de toutes les expériences réalisées pendant les années d'À la recherche $d u$ jazz. Cette synthèse jette pour longtemps les bases d'une forme d'art contemporain née de la télévision, qu'on appellera bientôt art vidéo, dont l'acte de naissance a été fixé par les historiens de l'art en mars 1963, lorsque Nam June Paik expose à la galerie Parnass de Wuppertal treize téléviseurs préparés pour la distorsion d'images. Mais, quelques années plus tôt, dans les studios de la RTF, Averty contribuait magistralement à la naissance imminente de cet art vidéo.

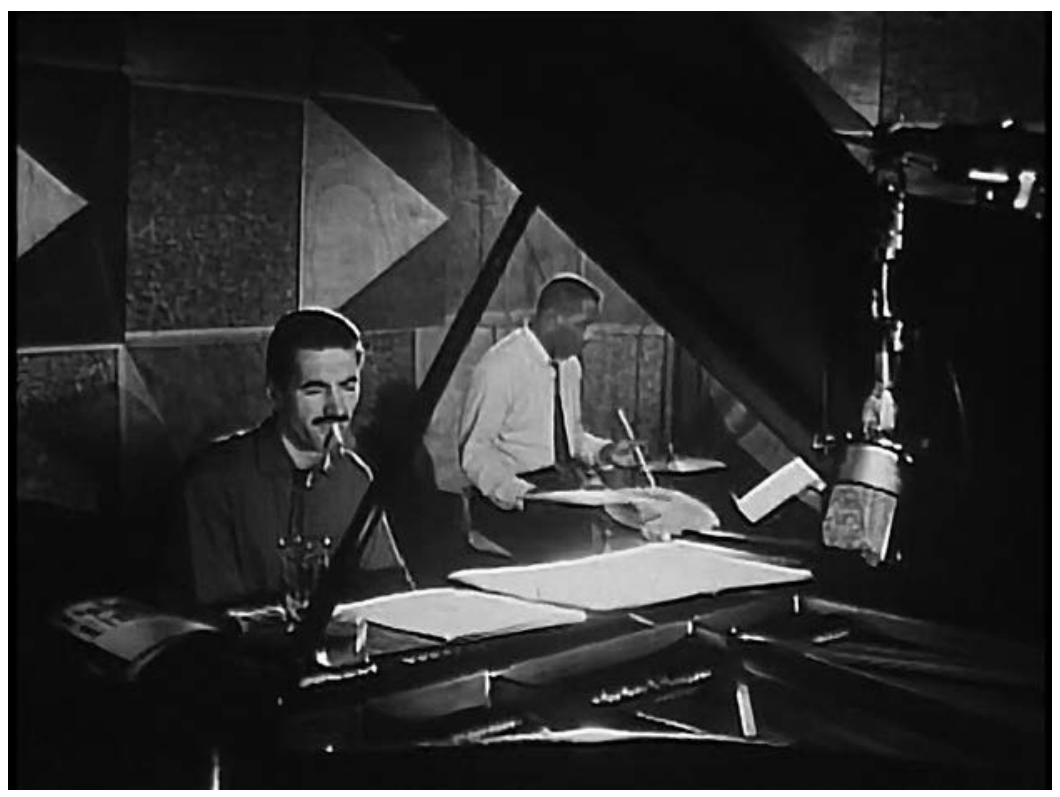

Fig. 6. Le dispositif technique, objet de toutes les attentions dans l'émission de la série $A$ la recherche du jazz intitulée "On enregistre" (Jean-Christophe Averty, 27 octobre 1957). 


\section{L'art d'Averty}

Fin connaisseur du jazz, honorable pianiste, Averty prend plaisir à imaginer émission après émission un langage spécifique qui s'enrichit à chaque diffusion de nouvelles propositions fondées sur une synergie tout à fait inédite entre les images de la télévision et une mobilité propre à l'improvisation jazziste. Si son goût personnel le porte vers le style New Orleans, il s'inspire surtout des audaces du be-bop pour concevoir des polyphonies et des polyrythmies de plus en plus complexes qui s'inscrivent au cœur des échanges virtuoses entre les solistes et les sections rythmiques. Il ne se contente pas de rendre compte de la vitalité collective du jazz: il le prend comme modèle pour inventer un langage fondé sur un dialogue en temps réel entre les sons et les images, sur une réactivité d'autant plus surprenante qu'elle dépend d'imposants prototypes qui ont pour noms truqueur, mélangeur ou incrustateur, expérimentés ici avec un goût du bricolage partagé par nombre de pionniers du nouveau média. Si cette batterie de trucages est assez modeste, Averty n'en parvient pas moins à créer un étonnant répertoire à partir d'utilisations peu orthodoxes de ces machines qu'il détourne souvent de leurs fonctions premières, ainsi que l'écrit Anne-Marie Duguet (1991, p. 118) dans la monographie qu'elle consacre à l'artiste:

La manière dont il explore les qualités de la matière électronique, tord, étire, disloque ses images, se réfère plutôt à [des] types de traitement sonore tels que le jazz les pratique, faisant grincer certains sons, les triturant, jouant d'inflexions et de vibratos.

Les effets dirty des musiciens de jazz qui éprouvent tout autant les possibilités de la voix humaine que les potentialités bruitistes de leurs instruments servent donc de modèles à Averty et à ses complices, attentifs aux défaillances des appareils ou aux branchements fortuits des techniciens: tout ici est source d'innovation.

Le modèle du jazz et de ses représentations est analysable à plusieurs niveaux dans le travail d'Averty. Alors que le gris envahit les décors de la télévision, Averty se sert aussi bien de la couleur de peau et des costumes noirs impeccables des musiciens que des touches du piano ou du vibraphone pour faire naître de violents contrastes de noir et de blanc, sources d'un nombre

Le jazz à la télévision française dans les années 1950 : du didactisme des premières représentations aux expérimentations de Jean-Christophe Averty 
infini de motifs qui renouvellent des trucages pourtant limités aux effets de fondu, de cut, de volet, de surimpression ou de négatif. Il gardera dans toutes ses aventures jazzistes la même volonté de créer dans les conditions du direct ou avec le goût du direct qui fait de lui un improvisateur impliqué dans le présent de la performance au même titre que les musiciens eux-mêmes. Il renouvelle les formes en jouant de ses effets avec une maittrise impressionnante de l'espace de l'écran de télévision, où la souplesse des corps et la géométrie des claviers ou des percussions semblent en dialogue permanent dans la profondeur de champ. C'est en plasticien, héritier de l'histoire de la peinture moderne et du cinéma, qu'Averty réinvente les équivalences entre le jazz et les effets visuels, dans un échange joyeux et modeste où les images sont toujours au service de la musique, où les effets spéciaux sont partie prenante de la polyphonie visuelle et sonore qui s'improvise sous nos yeux. "La musique est un nerf de l'image", selon la belle formule d'Anne-Marie Duguet (1991, p. 115), "jamais un accompagnement, comme l'image n'en est jamais la seule illustration ».

Les talents de musicien de jazz d'Averty constituent alors une clé essentielle pour comprendre cette osmose entre les sons et les images. Il sait parfaitement en quoi consiste cette particularité rythmique qu'on appelle le swing, principe vital du jazz, intraduisible en signes de solfège. "Le swing, écrit Jacques Réda (1988, p. 986), apparaît comme l'état supérieur, impossible à fixer mais puissant et irréfutable, de ce retournement du pas humain en danse que le jazz a produit ". L'implication des effets spéciaux d'Averty dans les images du jazz est de nature fondamentalement musicale: il sait pourquoi telle incrustation doit intervenir très légèrement en avance sur la phrase du soliste pour créer une syncope et intégrer la pulsation des musiciens, cet indéfinissable swing. Il peut ainsi multiplier les effets de défiguration sans perturber pour autant la continuité du mouvement si caractéristique de cette musique, la fluidité de ce swing, et réconcilier ainsi la photogénie du jazz telle que l'ont forgée les premières décennies du parlant et l'appétit d'enregistrement en direct si cher à la télévision. Avec les effets spéciaux du créateur de l'art vidéo qu'est Averty, ce n'est plus la musique qui est mise 


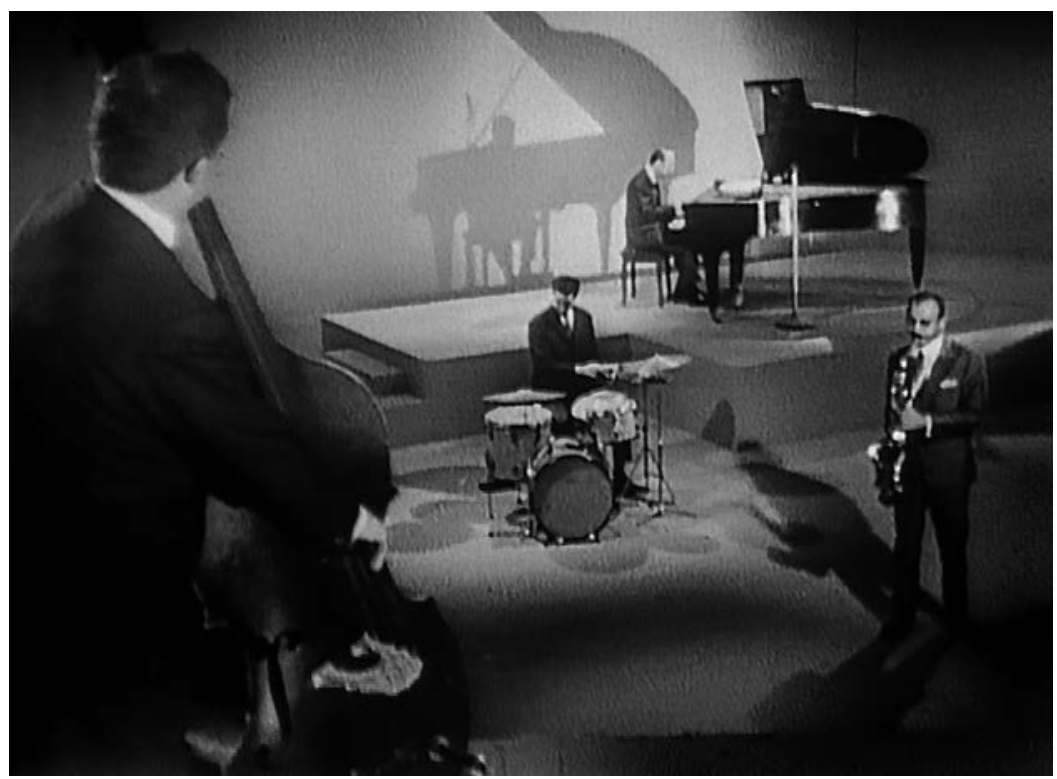

Fig. 7. La profondeur de champ dans Modern Jazz at Studio 4 (JeanChristophe Averty, 17 novembre 1960).

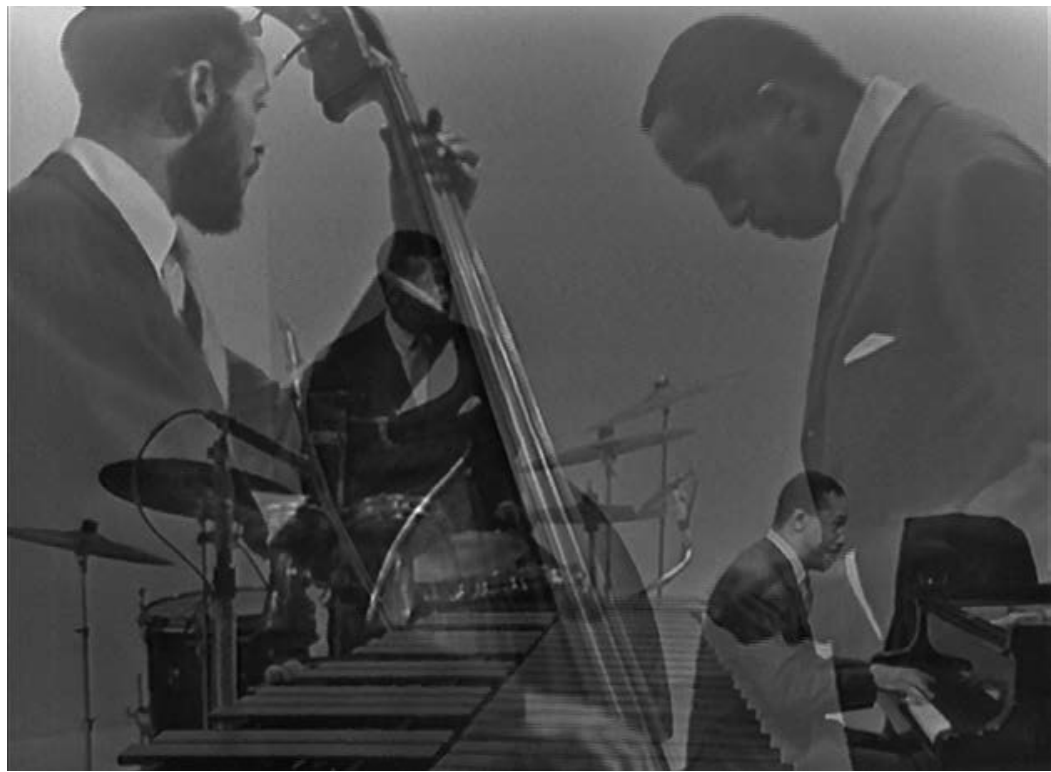

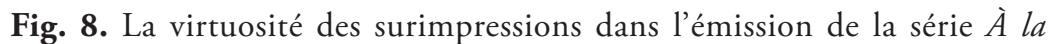
recherche du jazz intitulée "Modern Jazz Quartet» (Jean-Christophe Averty, 23 juillet 1957).

Le jazz à la télévision française dans les années 1950 : du didactisme des premières représentations aux expérimentations de Jean-Christophe Averty 
en images, mais l'improvisation collective dans son surgissement et sa complexité. Il n'aura de cesse que d'atteindre lui aussi l'immédiateté du geste improvisé pour parvenir, grâce à sa maîtrise des moyens spécifiques de la télévision, à faire swinguer les machines.

\section{Conclusion}

Le chemin parcouru entre $\grave{A}$ la recherche du jazz et Modern Jazz at Studio 4 est celui d'une double émancipation. Émancipation tout d'abord à l'égard des images du cinéma, quand les auteurs et réalisateurs de la télévision imaginent peu à peu, avec la maladresse et l'audace des pionniers, des modes de figuration inédits fondés sur de nouveaux rapports à la parole et à la transmission du savoir. Cette première émancipation a eu pour vertu de créer une formidable mémoire visuelle du jazz, mémoire dont seule une infime partie est aujourd'hui étudiée. Paroles des experts, paroles des jazzmen, mais surtout performances des créateurs majeurs de cette musique sont aujourd'hui accessibles dans les archives de la télévision ou sur Internet, traces inestimables de ces corps engagés tout entiers dans le temps du jeu, précieux témoignages de ce que fut alors le jazz. Outre les émissions conservées en France à l'Institut national de l'audiovisuel (INA), les télévisions américaine et anglaise ont aussi leur part de trésors avec, pour ne citer que quelques émissions, The Sound of Jazz, créée en 1957 pour la chaîne CBS par le producteur Robert Herridge, Jazz Casual de Ralph Gleason en 1961, Jazz Scene USA de Steve Allen en 1962 ou encore, en 1964, l'émission de la BBC Jazz 625, présentée en public par, entre autres, le trompettiste Humphrey Lyttelton.

La seconde émancipation, dont Jean-Christophe Averty est le plus illustre représentant, consiste à dépasser cette capacité à témoigner, à expliquer, à enregistrer, pour concevoir une discipline artistique inédite qui, à partir de nouveaux moyens d'expression, trouve sa place dans l'histoire de l'art $\mathrm{du} \mathrm{XX}^{\mathrm{e}}$ siècle. Pour inventer cet art vidéo, Averty est passé par le jazz, non seulement en s'inspirant des qualités propres à cette musique, mais en prenant en compte la diversité des rencontres entre le jazz et les autres arts, le cinéma bien sûr, qu'il soit d'animation ou en 


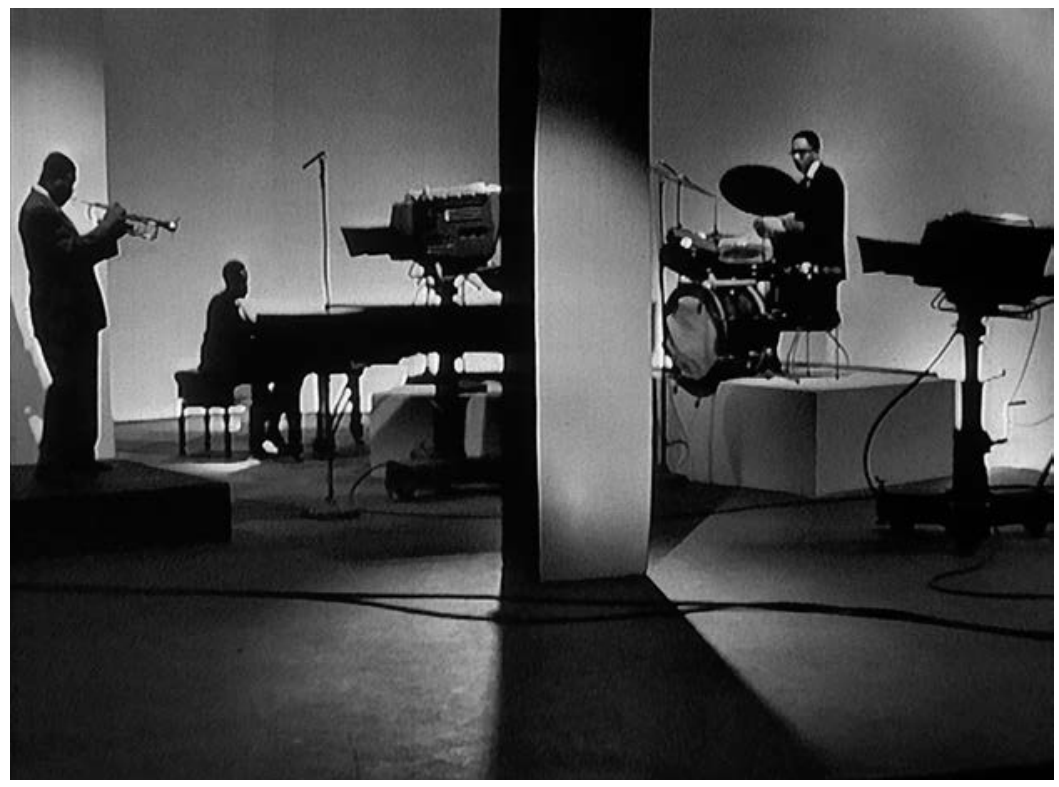

Fig. 9. L'espace et la technique dans Blue Note Parade (Jean-Christophe Averty, 6 avril 1963).

prises de vues réelles, mais aussi la danse, la poésie, la peinture ou les arts graphiques (il suffit de prêter attention aux génériques de ses émissions), tout cela réinvesti par les moyens techniques propres à la télévision. Cette fidélité à la télévision et au jazz est sans doute la raison essentielle de l'absence de reconnaissance de Jean-Christophe Averty dans le monde de l'art, mais elle lui a permis d'inventer le premier art populaire d'avantgarde, ce qui n'est pas rien!

Remerciements à l'INA Atlantique et notamment à Jean-Paul Dibouès.

Université Rennes 2

\section{NOTES}

1. Titre d'une émission de Yann Gill réalisée par André Hugues, avec notamment Sydney Bechet, Claude Luter et Roy Eldridge.

2. Avec Aimé Barelli et Hubert Rostaing; réalisée par Vicky Ivernel et diffusée le 21 novembre 1952.

Le jazz à la télévision française dans les années 1950: du didactisme des premières représentations aux expérimentations de Jean-Christophe Averty 
3. Blettery est le producteur et le présentateur de la série; il continuera une carrière de réalisateur pour la télévision.

4. Notons ici que dans les années 1950 des groupes de "puristes» soucieux de faire reconnaître par tous les moyens le "vrai jazz» multiplient les actions dans la presse ou à la radio, fustigeant les choix trop consensuels et pas assez radicaux de la première télévision; voir l'ouvrage de Ludovic Tournès (1999) sur l'histoire du jazz en France, notamment le chapitre VIII, intitulé "La conquête des médias".

5. En 1948, dix des quatorze heures de programme hebdomadaire sont constituées de longs métrages, pour la plupart récents, avant une régulation de la diffusion entre les salles et le petit écran qui interviendra dès l'année suivante; voir Sauvage et VeyratMasson 2012, p. 44.

6. La date exacte de la diffusion de cette émission est inconnue.

7. «Modern Jazz Quartet», 23 juillet 1957 (voir la figure 3); on notera le décor très Grand Siècle, destiné sans doute à affirmer la reconnaissance du jazz dans le panthéon des arts savants.

8. «Pourquoi le jazz?», 24 avril 1956.

9. Albert Pierru (1920-1983) a réalisé une vingtaine de courts métrages, dont un certain nombre de films peints sur pellicule, parmi lesquels Soir de fête (1956) et Surprise Boogie (1957), très influencés par les œuvres de Norman McLaren; la Cinémathèque française lui a rendu hommage le 11 janvier 2013. Voir également Gilles Mouëllic, "Albert Pierru et le jazz», communication au colloque Dessiner le jazz organisé les 20 et 21 novembre 2015 à Paris par le laboratoire SefeA de l'Université Sorbonne Nouvelle - Paris 3.

10. La date exacte de la diffusion de cette émission est inconnue.

11. Il semble que des extraits des émissions d'Averty aient été rediffusés dans des interludes dont le nom générique était "Télé music».

12. Voir Mouëllic 2014.

\section{RÉFÉRENCES BIBLIOGRAPHIQUES}

Delavaud 2003: Gilles Delavaud, "André Bazin, critique de télévision", dans Jérôme Bourdon et Jean-Michel Frodon (dir.), L'oil critique. Le journaliste critique de télévision, Paris/Bruxelles, INA/De Boeck, 2003, p 47-56.

Delavaud 2007: Gilles Delavaud, "Penser la télévision avec le cinéma", Cinémas, vol. 17, ${ }^{\text {os }} 2-3,2007$, p. 73-95.

Duguet 1991 : Anne-Marie Duguet, Jean-Christophe Averty, Paris, Dis Voir, 1991.

Lavat 2011 : Philippe Lavat, "L’apparition du jazz à la télévision", dans Sylvie Pierre (dir.), La télévision, le temps des constructeurs. Jean d'Arcy, pensée et stratégies d'un père fondateur, Paris, L'Harmattan, 2011, p. 73-80.

Mouëllic 2014: Gilles Mouëllic, "La télévision comme dispositif d'enregistrement du jazz», dans Pierre-Henry Frangne et Hervé Lacombe (dir.), Musique et enregistrement, Rennes, Presses universitaires de Rennes, 2014, p. 259-267.

Réda 1988: Jacques Réda, article "Swing» dans Philippe Carles, André Clergeat et Jean-Louis Comolli (dir.), Dictionnaire du jazz, Paris, Robert Laffont, 1988, p. 986.

Sauvage et Veyrat-Masson 2012 : Monique Sauvage et Isabelle Veyrat-Masson, avec la collaboration de Géraldine Poels, Histoire de la télévision française de 1935 à nos jours, Paris, Nouveau Monde, 2012.

Tournès 1999: Ludovic Tournès, New Orleans sur Seine. Histoire du jazz en France, Paris, Fayard, 1999. 


\section{ABSTRACT}

\section{Jazz on French Television in the 1950s: From the Didacticism of the Early Years to the Experimentation of Jean-Christophe Averty \\ Gilles Mouëllic}

Jazz had a prominent place on French radio and in the French press after the Second World War, and gradually took its place on French television after 1955. The vast majority of the programs making up the first regular series on jazz, $\dot{A}$ la recherche $d u$ jazz, created by Maurice Blettery, were directed by JeanChristophe Averty. They reveal a tension between two didactic ambitions: one used jazz as a pretext to address "social topics," while the other sought to explore music for its own sake and to espouse jazz as the sign of a new equilibrium between "highbrow" and "popular" culture. Although the earliest programs were constructed around an often awkward alternation of clips from feature films and remarks by acknowledged experts, Averty gradually broke free of the series' editorial constraints to set the music to images and explore the resources television offered. In the late 1950s, he took over new programs such as Modern Jazz at Studio 4, in which he featured musical performances and made jazz an ideal locus of experimentation for inventing a visual world which we might describe, long before the work of Nam June Paik, as video art.

Le jazz à la télévision française dans les années 1950 : du didactisme des premières représentations aux expérimentations de Jean-Christophe Averty 